\title{
Undoing the twist: the Hořava limit of Einstein-aether
}

\author{
Ted Jacobson \\ Center for Fundamental Physics, University of Maryland, College Park, MD 20742-4111, USA. \\ Institut d'Astrophysique de Paris, 98bis bv. Arago, 75014 Paris, France.
}

(Dated: September 21, 2018)

\begin{abstract}
We show that Hořava gravity can be obtained from Einstein-aether theory in the limit that the twist coupling constant goes to infinity, while holding fixed the expansion, shear and acceleration couplings. This limit helps to clarify the relation between the two theories, and allows Hořava results to be obtained from Einstein-aether ones. The limit is illustrated with several examples, including rotating black hole equations, PPN parameters, and radiation rates from binary systems.
\end{abstract}

PACS numbers: $04.50 . \mathrm{Kd}, 04.20 . \mathrm{Fy}$

\section{INTRODUCTION}

Einstein-aether theory $[1-3]$ is a generally covariant modification of general relativity (GR), in which the spacetime metric $g_{a b}$ is coupled to a unit timelike vector field $u^{a}$, the "aether". Hořava gravity $[4,5]$ is a related theory, in which the aether is kinematically restricted at the level of the action to be twist-free or, equivalently, hypersurface orthogonal. Originally formulated in terms of the spatial metric, lapse and shift on a fixed foliation, Hořava gravity includes higher spatial derivative terms leading to "Lifshitz scaling" at short distances, that render the theory power counting renormalizable as a quantum field theory. It is not clear whether a similar Lifshitz version can be formulated for Einstein-aether theory, since it lacks the preferred hypersurfaces that play a role in defining the scaling behavior. Here I will restrict attention to the IR limits of these theories, although if a Lifshitz extension of Einstein-aether theory exists, the main result of the paper would generalize to that context.

At second order in derivatives, the Einstein-aether (hereafter called "æ-theory") Lagrangian is a combination of five scalars (including the Ricci scalar $R$ ) that yield independent contributions to the equations of motion. The second order lagrangian for Hořava gravity (hereafter called "T-theory", and which is also known as Khronometric gravity [6]) consists of the same scalars, although the hypersurface-orthogonal (HO) condition reduces the number of independent ones from five to four $[6,7]$. Any $\mathrm{HO}$ solution to the æ-theory field equations is also a $T$-theory solution, since the action is stationary with respect to all variations of the aether (preserving unit norm), not just HO variations [7]. For the same reason, some $T$-theory solutions are not æ-theory solutions. An example is provided by the (perturbative) rotating black hole solutions [9-12].

The main purpose of this paper is to identify a limit of æ-theory that coincides with $T$-theory. This limit includes not only the HO solutions of æ-theory, but also $T$-theory solutions that are not æ-theory solutions, for example the rotating black hole solution just mentioned. I have not quite proved that all $T$-theory solutions arise via this limit, but I will argue that, if they do not, it is only because of boundary effects. Having established this limit, the relation between the theories can be better understood, and information about $T$-theory can be obtained using results previously established for Einsteinaether theory.

In brief, the argument goes as follows. One of the five independent terms in the Lagrangian is chosen to be the square of the twist of the aether (to be defined explicitly below), which vanishes if and only if the aether is HO. The coupling coefficient of this term is sent to infinity, so that the twist terms in the aether and metric equations of motion diverge unless they vanish. In this limit, a solution can remain regular only if all of these terms vanish; but not all of them can vanish unless the twist itself vanishes. This limit therefore selects the HO Einstein-aether solutions. More surprisingly, the limit has the effect of suppressing the terms in the field equation that come from non-HO variations of the metric, so one obtains also $T$-theory solutions that are not æ-theory solutions. This phenomenon is explained in Sec. III, and illustrated in Sec. III A using the rotating black hole solution. In Sec. III B various parameters characterizing the physics of $T$-theory are derived from those of æ-theory by use of this limit, and Sec. III C discusses how the difference in PPN parameters affects the observational constraints.

\section{II. Æ-THEORY AND T-THEORY}

The two-derivative action for æ-theory has the form

$$
S=\frac{1}{16 \pi G_{0}} \int\left(-R+L_{æ}\right) \sqrt{-g} d^{4} x
$$

where $R$ is the $4 \mathrm{D}$ Ricci scalar, the aether Lagrangian $L_{æ}$ is a sum of four independent scalars quadratic in $\nabla_{a} u^{b}$, and the unit constraint $g_{a b} u^{a} u^{b}=1$ is either implicit or imposed with a Lagrange multiplier term. (The conventions are those of [13] except for the spacetime signature, which is taken as $(+---)$.) In most of the literature, the aether Lagrangian has been expressed as $-\left(c_{1} L_{1}+c_{2} L_{2}+c_{3} L_{3}+c_{4} L_{4}\right)$, where the $c_{i}$ 's are dimen- 
sionless coupling constants, and

$$
\begin{aligned}
& L_{1}=\left(\nabla_{a} u_{b}\right)\left(\nabla^{a} u^{b}\right) \\
& L_{2}=\left(\nabla_{a} u^{a}\right)^{2} \\
& L_{3}=\left(\nabla_{a} u_{b}\right)\left(\nabla^{b} u^{a}\right) \\
& L_{4}=\left(u^{m} \nabla_{m} u^{a}\right)\left(u^{n} \nabla_{n} u_{a}\right) .
\end{aligned}
$$

It is more revealing, however, to use a decomposition of the covariant derivative of the aether into terms transforming by irreducible representations of the $S O(3)$ Lorentz subgroup that leaves the aether invariant,

$$
\nabla_{a} u_{b}=-\frac{1}{3} \theta h_{a b}+\sigma_{a b}+\omega_{a b}+u_{a} a_{b} .
$$

Taking into account the unit norm of $u^{a}$, the spatial projection of $\nabla_{a} u_{b}$ is $\nabla_{a} u_{b}-u_{a} a_{b}$, where $a^{a}=u^{m} \nabla_{m} u^{a}$ is the acceleration (which is orthogonal to $u^{a}$ ). The expansion term $-\frac{1}{3} \theta h_{a b}$ and shear $\sigma_{a b}$ are the trace and trace-free parts of the symmetric part of this spatial projection, while the twist $\omega_{a b}$ is the anti-symmetric part,

$$
\omega_{a b}=\nabla_{[a} u_{b]}-u_{[a} a_{b]} .
$$

(A different quantity that contains essentially the same information, and is also called the twist, is $\tilde{\omega}^{a} \equiv$ $\epsilon^{a b c d} u_{b} \nabla_{c} u_{d}=\epsilon^{a b c d} u_{b} \omega_{c d}$.) The twist vanishes if and only if $u_{[a} \nabla_{b} u_{c]}$ vanishes. If the aether is $\mathrm{HO}, u_{a}$ can be written as $u_{a}=f \nabla_{a} g$ for some functions $f$ and $g$, hence $u_{[a} \nabla_{b} u_{c]}=f \nabla_{[a} g \nabla_{b} f \nabla_{c]} g=0$, so the twist vanishes. That the converse holds is not so obvious, but it is implied by the Frobenius theorem [13].

The four terms in (6) are mutually orthogonal with respect to the spacetime metric $g_{a b}$, the first three have zero contraction with $u^{a}$ on either index, the last three have zero contraction with $g^{a b}$, so the only quadratic scalars that can be formed from them using $g_{a b}$ and $u^{a}$ are just their squares formed with the metric. These squares can serve as the scalars defining the Lagrangian. The action for Einstein-aether theory can thus be written as

$S=\frac{-1}{16 \pi G_{0}} \int\left(R+\frac{1}{3} c_{\theta} \theta^{2}+c_{\sigma} \sigma^{2}+c_{\omega} \omega^{2}+c_{a} a^{2}\right) \sqrt{-g} d^{4} x$.

The relation between the coupling constants defined here and those that have been used previously for æ-theory is found by substitution of the decomposition (6) in (2-5), which yields

$$
\begin{aligned}
c_{\theta} & =c_{1}+c_{3}+3 c_{2} \\
c_{\sigma} & =c_{1}+c_{3} \\
c_{\omega} & =c_{1}-c_{3} \\
c_{a} & =c_{1}+c_{4} .
\end{aligned}
$$

These combinations of coupling constants have been found to appear in numerous physical quantities [3].

The aether in æ-theory is unconstrained, other than being a unit vector, so it has three kinematic degrees of freedom. In $T$-theory the aether is restricted to be $\mathrm{HO}$, so it can be expressed in terms of the gradient of a scalar field $T$ (the "khronon" [6]), via

$$
u^{a}=\frac{\nabla^{a} T}{\sqrt{\left(\nabla_{m} T\right)\left(\nabla^{m} T\right)}} .
$$

(Note that $u^{a}$ is unchanged if $T$ is replaced by any monotonically increasing function of $T$.) The action for $T$ theory is the same as the æ-theory action (8), but with the aether defined by $(13)[6,7]$. The twist term vanishes identically for $\mathrm{HO} u^{a}$, however, so the coupling constant $c_{\omega}$ plays no role. A common notation for the remaining constants $c_{a}, c_{\sigma}$, and $c_{\theta}$ in $T$-theory is $\alpha, \beta$, and $\beta+3 \lambda$, respectively [8]. Hořava's original formulation results by choosing $T$ as a spacetime coordinate, thus fixing some of the coordinate freedom. This coordinate choice may be imposed in the action, since the $T$ equation of motion is implied by the other equations of motion [7].

\section{A. Remarks on the form of the action}

As an aside, it is worth making a few remarks about the action (8). This action was first constructed in Ref. [14] using the irreducible parts of the covariant derivative of the Goldstone bosons associated with the breaking of local Lorentz invariance down to the rotation group. The authors also noted that the coupling constants of the irreducible terms are the ones that appear more simply in many physical properties of the theory. Indeed, with the action expressed in this way, it is sometimes easy to see which terms, and hence which coupling constants, can play a role in a given setting. For instance, in a static configuration, the aether when tangent to a timelike Killing vector has vanishing expansion, shear, and twist. Since these quantities appear quadratically in the action, those terms cannot contribute, so only the acceleration coupling $c_{a}$ enters such solutions. As the Newtonian limit is based on such solutions, the value of Newton's constant $G_{N}$ (22) also depends only on $c_{a}$. Similarly, in homogeneous, isotropic configurations the aether has vanishing shear, twist, and acceleration, so such solutions, as well as the cosmological gravitational constant $G_{\text {cosmo }}(23)$, involve only the expansion coupling $c_{\theta}$. As to waves, the spin (helicity) content of the various quantities is: expansion $(0)$, shear $(2,1,0)$, twist $(1)$, and acceleration $(1,0)$. Hence the spin-2 wave speed involves only $c_{\sigma}$, the spin-1 wave speed involves all but $c_{\theta}$, and the spin- 0 wave speed involves all but $c_{\omega}$.

\section{T-THEORY LIMIT OF Æ-THEORY}

Now consider the $c_{\omega} \rightarrow \infty$ limit of $æ$-theory, with all other coupling constants held fixed. ${ }^{1}$ (In terms of the

\footnotetext{
1 A similar method was used in Ref. [5] to relate the projectable case of Hořava gravity (lapse function spatially constant) to the
} 
usual parameters, this limit corresponds to $c_{1}-c_{3} \rightarrow \infty$, with $c_{1}+c_{3}, c_{1}+c_{4}$, and $c_{2}$ held fixed. Thus $c_{1}, c_{3}$, and $c_{4}$ all diverge in this limit.) Any term in the equations of motion involving $c_{\omega}$ will diverge in this limit unless that term vanishes. One of these terms, which arises from varying the volume element, is $\sim c_{\omega} \omega^{2} g_{a b}$, and the only way this can vanish is if $\omega_{a b}=0$. Thus we may conclude that only $\mathrm{HO}$ solutions are regular in this limit. However, if the limit is to coincide with $T$-theory, then not only $æ$-theory solutions but all $T$-theory solutions should arise in the limit. This would be the case only if the limit somehow "turned off" the part of the aether field equations that arises from non-HO variations of the action. This seems indeed to be the case, except for possible boundary effects.

To understand how this works, it is instructive to consider an elementary analogy, in which the action $S(x, y)$ depends on just two variables $x$ and $y$, with $y$ playing the role of the twist. Expanding in $y$, we have

$$
S(x, y)=S_{0}(x)+S_{1}(x) y+c_{y} S_{2}(x) y^{2}+\ldots,
$$

where $c_{y}$ is a coupling parameter, analogous to $c_{\omega}$, that we will take to infinity. Dropping for the moment any higher order terms in $y$, the $y$ and $x$ equations of motion are given, respectively, by

$$
\begin{aligned}
S_{1}(x)+2 c_{y} S_{2}(x) y & =0 \\
S_{0}^{\prime}(x)+S_{1}^{\prime}(x) y+c_{y} S_{2}^{\prime}(x) y^{2} & =0 .
\end{aligned}
$$

Unless $S_{2}(x)$ vanishes, the only regular solutions in the limit $c_{y} \rightarrow \infty$ will have $y=0$. If we set $y$ to zero before taking the limit, then the $y$ equation (15) survives as $S_{1}(x)=0$. However, we could also solve the $y$ equation for every finite $c_{y}$, obtaining $y=-S_{1}(x) /\left(2 c_{y} S_{2}(x)\right)$. In the limit, we then have $y=0$, and the $y$ equation has been satisfied without imposing any further conditions on $x$. If $S(x, y)$ contains higher order terms in $y$ that are independent of $c_{y}$, then still the only regular solutions in the limit will be those with $y=0$, and still the $y$ equation will imply nothing about $x$.

This simple example can be generalized to any finite number of coordinates, and even to a field theory setting. However, when the variables are fields and the action involves derivatives, integration by parts can lead to boundary terms. Boundary terms could be avoided by working on a compact space, or by fixing boundary conditions. I therefore expect the argument should hold locally for æ-theory, although in a non-compact space some $T$-theory solutions might perhaps be missing from the limit.

\section{A. Rotating black hole}

An example of the appearance, in the limit, of $T$-theory solutions that are not æ-theory solutions, occurs in the

non-projectable case, taking the limit $c_{a} \rightarrow \infty$. case of the slowly rotating black holes. There are no HO æ-theory solutions $[9,11]$, but there are $T$-theory solutions of this type [9-12]. We can see them arise in the $c_{\omega} \rightarrow \infty$ limit, making use of the field equations in Ref. [15], which include the first order contributions of the rotation.

Consider in particular equations (60) and (61) of that paper, which hold in æ-theory under the assumption that the spacetime is asymptotically flat:

$$
\begin{aligned}
d_{1} \psi^{\prime}+d_{2} \psi^{\prime \prime}+d_{3} \lambda^{\prime}+d_{4} \lambda^{\prime \prime} & =0 \\
b_{1} \psi^{\prime}+b_{2} \psi^{\prime \prime}+b_{3} \lambda^{\prime}+b_{4} \lambda^{\prime \prime} & =-r^{-4} \lambda,
\end{aligned}
$$

where $\psi, \lambda$ and all the coefficients are functions of $r$. The coefficient functions depend on the background spherical black hole solution and the coupling constants. The function $\psi(r)$ determines the angular velocity of the aether, while the function $\lambda(r)$ governs the twist, in the sense that the twist vanishes if and only if $\lambda(r)=0$. It has been shown that asymptotic flatness requires $\lambda \rightarrow 0$ as $r \rightarrow \infty[16]$.

If the aether is $\mathrm{HO}$, so that $\lambda(r)=0$, then by combining the two equations one finds that $\psi^{\prime}(r)=0$, which implies that the solution must be the non-rotating black hole (generally in rotating coordinates) [11]. That is, there is no HO rotating black hole solution in æ-theory. In $T$-theory, on the other hand, Eq. (17) is not present, and the general solution to Eq. (18) for $\psi(r)$ describes a rotating black hole.

Consider now what happens if $\lambda(r)$ is kept non-zero in the æ-theory equations as the limit is taken. The functions $b_{1,2,3,4}$ are independent of $c_{\omega}$, the functions $d_{1,2,4}$ diverge as $c_{\omega}$ in the limit, and the function $d_{3}$ diverges as $c_{\omega}^{2}$. Therefore (17) can be viewed as an equation for $\lambda(r)$ which implies that $\lambda^{\prime}(r)=0$ in the limit. Since all asymptotically flat æ-theory solutions have $\lambda(\infty)=0$, this implies $\lambda(r)=0$. The remaining equation thus reduces in the limit to the $T$-theory equation, and so the $T$-theory solutions are recovered in the limit.

\section{B. T-theory parameters from æ-theory ones}

Quantities characterizing $T$-theory can be obtained as the infinite $c_{\omega}$ limit of those characterizing æ-theory. In this section a number of such quantities will be examined, both as an illustration of the correspondence, and to elucidate the relation between the two theories.

To begin with, the wave speeds $s_{2,1,0}$ for the spin-2, spin- 1 , and spin- 0 waves in $æ$-theory are given by [17]

$$
\begin{aligned}
& s_{2}^{2}=\frac{1}{1-c_{\sigma}} \\
& s_{1}^{2}=\frac{\left.c_{\sigma}+c_{\omega}-c_{\sigma} c_{\omega}\right)}{2 c_{a}\left(1-c_{\sigma}\right)} \\
& s_{0}^{2}=\frac{\left(c_{\theta}+2 c_{\sigma}\right)\left(1-c_{a} / 2\right)}{3 c_{a}\left(1-c_{\sigma}\right)\left(1+c_{\theta} / 2\right)} .
\end{aligned}
$$


The spin-2 and spin-0 wave speeds are independent of $c_{\omega}$ so they coincide in the two theories, while the spin-1 wave speed diverges as $\sqrt{c_{\omega}}$. The energy density at fixed amplitude and frequency remains finite [18, 19], so the energy flux from a periodic source behaves as $\sqrt{c_{\omega}}$ times the squared wave amplitude. One can see by inspection of the radiation analysis of Ref. [19] that the wave amplitude generated by such a source vanishes as $c_{\omega}^{-3 / 2}$, so we conclude that the power radiated in spin-1 waves vanishes as $c_{\omega}^{-5 / 2}$. The spin-1 mode thus decouples in this limit.

Newton's constant $G_{N}$ and the cosmological gravitational constant $G_{\text {cosmo }}$ appearing in the Friedmann equation are related to the "bare" constant $G$ in the action (8) by $[20]$

$$
\begin{aligned}
G_{N} & =G /\left(1-c_{a} / 2\right) \\
G_{\text {cosmo }} & =G /\left(1+c_{\theta} / 2\right) .
\end{aligned}
$$

Both of these relations are independent of $c_{\omega}$, hence they hold also in T-theory, as shown independently in Ref. [5].

The PPN parameters are also related by this limit. All PPN parameters of ae-theory are identical to those of GR, except for the preferred frame parameters $\alpha_{1}$ and $\alpha_{2}$, which are given by $[21,22]$

$$
\begin{aligned}
\alpha_{1} & =4 \frac{c_{\omega}\left(c_{a}-2 c_{\sigma}\right)+c_{a} c_{\sigma}}{c_{\omega}\left(c_{\sigma}-1\right)-c_{\sigma}} \\
\alpha_{2} & =\frac{\alpha_{1}}{2}+\frac{3\left(c_{a}-2 c_{\sigma}\right)\left(c_{\theta}+c_{a}\right)}{\left(2-c_{a}\right)\left(c_{\theta}+2 c_{\sigma}\right)} .
\end{aligned}
$$

In the infinite $c_{\omega}$ limit $\alpha_{1}$ becomes

$$
\alpha_{1}^{T}=4 \frac{c_{a}-2 c_{\sigma}}{c_{\sigma}-1},
$$

and the formula (25) for $\alpha_{2}$ is otherwise unchanged, in agreement with previous computations for $T$-theory $[6$, 8].

Finally, radiation amplitudes and rates can be carried over in the limit from æ-theory to $T$-theory, both in the weak and strong self-gravity regimes. For weak self-gravity, the results of [8] are obtained from the limit of those of [19] (modulo typos), while for strong selfgravity, the limit of [23] should agree with new results for $T$-theory, which have recently been derived $a b$ initio in [24] and reported in [25]. (In fact, computational errors in [23] were discovered by the failure of the limit to agree.)

\section{Observational constraints}

Equations (24-26) reveal an important discrepancy in how $\alpha_{1}$ and $\alpha_{2}$ can be set to zero (or to small values) in the two theories [8]. In æ-theory two conditions on the parameters are required [22]:

$$
\begin{aligned}
& c_{a}=2 \frac{c_{\omega} c_{\sigma}}{c_{\sigma}+c_{\omega}} \longrightarrow 2 c_{\sigma} \\
& c_{\theta}=-c_{a}=-2 \frac{c_{\omega} c_{\sigma}}{c_{\omega}+c_{\sigma}} \longrightarrow-2 c_{\sigma},
\end{aligned}
$$

where the arrows denote the limit $c_{\omega} \rightarrow \infty$. (An alternative would be to set $c_{a}=0=c_{\sigma}$, which would make the spin-0 and spin-1 wave speeds diverge.) In $T$-theory the first condition (27) alone, $c_{a}=2 c_{\sigma}$, suffices to set both $\alpha_{1}^{T}$ and $\alpha_{2}^{T}$ to zero. (Since there is one less coupling constant to begin with in $T$-theory, this again leaves a two-dimensional coupling constant space unconstrained, as in æ-theory.) Hence the second condition, (28), need not be applied, so the limit of the æ-theory conditions for the vanishing of the PPN parameters is stronger than the $T$-theory condition.

On the other hand, this second condition (28) for ætheory implies also that $G_{N}=G_{\text {cosmo }}(22,23)$, ensuring agreement with the primordial nucleosynthesis constraint [20] and constraints from the spectrum of CMB and matter anisotropies [26]. If the PPN constraints are met in $T$-theory just by imposing the first condition, $c_{a}=2 c_{\sigma}$, then these cosmological constraints must be separately imposed as the requirement that the second condition hold approximately, up to a deviation smaller than something of order $\sim 0.1$ for nucleosynthesis [8] and $\sim 0.01$ for anisotropies [26].

If exact vanishing of $\alpha_{1,2}$ is imposed in $T$-theory, then $G_{\text {cosmo }} / G_{N}=\left(1-c_{\sigma}\right) /\left(1+c_{\theta} / 2\right)$, from which it follows that the spin-0 mode speed (21) vanishes as $G_{\text {cosmo }}$ approaches $G_{N}$. This is ruled out by the vacuum Cerenkov constraint [27] (arising from the observation of ultra-high energy cosmic rays) which requires the mode speed to be at least that of light (minus a tiny amount). If the spin-0 mode speed is adjusted to be unity, i.e. the minimum value allowed by the Cerenkov constraint, then it follows that $G_{\text {cosmo }} / G_{N}=1-3 c_{a} / 2$, so the nucleosynthesis constraint $\left|G_{\text {cosmo }} / G_{N}-1\right| \lesssim 0.13$ implies $c_{a} \lesssim 0.08$, i.e. $c_{\sigma} \equiv \beta \lesssim 0.04$. At the other extreme, as $c_{a}=2 c_{\sigma} \rightarrow 0$, the spin-0 mode speed diverges, $G_{\text {cosmo }} / G_{N}=1 /\left(1+c_{\theta} / 2\right)$, and the nucleosynthesis constraint implies $c_{\theta} \equiv 3 \lambda \lesssim 0.30\left(c_{\theta}=3 \lambda\right.$ when $\left.c_{\sigma}=0\right)$. These constraints are consistent with those reported in [25].

It was found in [8] that a qualitative difference in the scalar radiation for the two theories arises, again due to the different way the PPN parameters are set to zero. When restricting to $\alpha_{1,2}=0$, radiation sourced by the second time derivative of the second monopole moment $\left(\sim \int d^{3} r \ddot{\rho} r^{2}\right)$ vanishes in $æ$-theory but not in $T$-theory. The coupling to this monopole term is proportional to

$$
\frac{\alpha_{1}-2 \alpha_{2}}{c_{a}-2 c_{\sigma}}=\frac{6\left(c_{\theta}+c_{a}\right)}{\left(c_{a}-2\right)\left(c_{\theta}+2 c_{\sigma}\right)} .
$$

As explained above, in æ-theory the vanishing of the PPN parameters does not require $c_{a}=2 c_{\sigma}$ (but does entail $\left.c_{a}+c_{\theta}=0\right)$, so the monopole coupling vanishes. In $T$-theory, the vanishing of the PPN parameters requires $c_{a}=2 c_{\sigma}$ only, so the left hand side of $(29)$ is $0 / 0$, and the right hand side shows that the coupling survives. 


\section{ACKNOWLEDGMENTS}

I am grateful to E. Barausse, D. Blas, T. Sotiriou and A. Speranza for helpful discussions, comments, and suggestions on a draft of this paper, and to the Institut
d'Astrophysique de Paris where its preparation was completed. This research was stimulated and informed by the Kavli IPMU workshop, "Gravity and Lorentz violations", and was supported in part by the NSF under grant No. PHY-0903572.
[1] See, for example, M. Gasperini, "Singularity prevention and broken Lorentz symmetry," Class. Quantum Grav. 4, 485 (1987); "Repulsive gravity in the very early Universe," Gen. Rel. Grav. 30, 1703 (1998); and references therein.

[2] T. Jacobson and D. Mattingly, Phys. Rev. D 64, 024028 (2001) [arXiv:gr-qc/0007031].

[3] T. Jacobson, PoS QG-PH, 020 (2007) [arXiv:0801.1547].

[4] P. Horava, Phys. Rev. D 79, 084008 (2009). [arXiv:0901.3775 [hep-th]].

[5] D. Blas, O. Pujolas and S. Sibiryakov, Phys. Rev. Lett. 104, 181302 (2010) [arXiv:0909.3525 [hep-th]].

[6] D. Blas, O. Pujolas and S. Sibiryakov, JHEP 1104, 018 (2011) [arXiv:1007.3503 [hep-th]].

[7] T. Jacobson, Phys. Rev. D 81, 101502 (2010) [Erratumibid. D 82, 129901 (2010)] [arXiv:1001.4823 [hep-th]].

[8] D. Blas and H. Sanctuary, Phys. Rev. D 84, 064004 (2011) [arXiv:1105.5149 [gr-qc]].

[9] E. Barausse and T. P. Sotiriou, Phys. Rev. Lett. 109, 181101 (2012) [Erratum-ibid. 110, 039902 (2013)] [arXiv:1207.6370].

[10] A. Wang, arXiv:1212.1040 [hep-th].

[11] E. Barausse and T. P. Sotiriou, Phys. Rev. D 87, 087504 (2013) [arXiv:1212.1334].

[12] A. Wang, Phys. Rev. Lett. 110, 091101 (2013) [arXiv:1212.1876].

[13] R.M. Wald, General Relativity (Univerity of Chicago Press, 2010).
[14] C. Armendariz-Picon, A. Diez-Tejedor and R. Penco, JHEP 1010, 079 (2010) [arXiv:1004.5596 [hep-ph]].

[15] E. Barausse and T. P. Sotiriou, arXiv:1307.3359 [gr-qc].

[16] E. Barausse and T. P. Sotiriou, private comunication.

[17] T. Jacobson and D. Mattingly, Phys. Rev. D 70, 024003 (2004) [gr-qc/0402005].

[18] C. Eling, Phys. Rev. D 73, 084026 (2006) [Erratum-ibid. D 80, 129905 (2009)] [gr-qc/0507059].

[19] B. Z. Foster, Phys. Rev. D 73, 104012 (2006) [Erratumibid. D 75, 129904 (2007)] [gr-qc/0602004].

[20] S. M. Carroll and E. A. Lim, Phys. Rev. D 70, 123525 (2004) [arXiv:hep-th/0407149].

[21] M. L. Graesser, A. Jenkins and M. B. Wise, Phys. Lett. B 613, 5 (2005) [hep-th/0501223].

[22] B. Z. Foster and T. Jacobson, Phys. Rev. D 73, 064015 (2006) [gr-qc/0509083].

[23] B. Z. Foster, Phys. Rev. D 76, 084033 (2007) [arXiv:0706.0704 [gr-qc]].

[24] K. Yagi, D. Blas, E. Barausse and N. Yunes, arXiv:1311.7144 [gr-qc].

[25] K. Yagi, D. Blas, N. Yunes and E. Barausse, arXiv:1307.6219 [gr-qc].

[26] B. Audren, D. Blas, J. Lesgourgues and S. Sibiryakov, JCAP 1308, 039 (2013) [arXiv:1305.0009 [astro-ph.CO], arXiv:1305.0009].

[27] J. W. Elliott, G. D. Moore and H. Stoica, JHEP 0508, 066 (2005) [hep-ph/0505211]. 